Original Contribution

\title{
PHYSIOTHERAPY FOR CONTROLLING THE COMPENSATORY MECHANISMS AFTER SURGICALLY TREATED COMPLEX ACETABULAR FRACTURES
}

\author{
R. Tasheva* \\ Physical Therapy Department - Theory and Methodology of the Physical Therapy, \\ National Sports Academy "Vassil Levski"- Sofia, Bulgaria
}

\begin{abstract}
The social relevance of the topic is the opportunity to prevent by physiotherapy the compensations that lead to complications which affect patients' daily lives. THE PURPOSE of this study is to present the physiotherapy to control the most common compensatory syndromes, namely "short leg", in patients after surgically treated complex acetabular fractures. METHOD Twenty two patients were operated on surgical stabilized method with reconstructive plates and screws from 2000 to 2019. Pelvis stabilization was achieved by physiotherapy modalities such as unilateral relaxation of hamstrings, erector spinae, multifidus and quadratus lumborum, and their contralateral stimulation. The patients were educated to control pelvis, to flex knee, to strike and rock the heel. RESULTS There were differences between greater trochanter-to-ankle and spina iliaca anterior superior-to-ankle measurements with average 1, 7 $\mathrm{cm}$. Were found the unilateral shorten muscles. The results of the tests were negative at the end of the relative protection phase for 7 procedures. CONCLUSION The social importance of physiotherapy is not only to restore correct and optimal patients, but also to provide prevention of complications after surgically stabilized complex acetabular fractures through avoid the compensatory mechanisms as a "short leg" syndrome.
\end{abstract}

Key words: acetabular fractures, physiotherapy, "short leg" syndrome

\section{INTRODUCTION}

The social relevance of the topic is determined in two directions. On the one hand, it is the increasing frequency of road accidents and on the other is the life-long compensations that lead to complications and affect patients' daily lives.

The acetabular fractures are included into the group of highly-energetic traumas caused by the most dangerous road crashes with cars, bikes, motorcycle and sports like motoring and hang gliding. In fact the surgical stabilization is a preferable treatment of dislocated fractures in the last years (2-4). The several authors reported some functional and postural problems and their arising without any correct observation and physiotherapy of the patients $(1,5,6,10)$.

\footnotetext{
Correspondence to: Rumiana Tasheva, 1 Gurguliat, Sofia, National Sports Academy, Tel: 08933965 83; 0888559107, e-mail: rumiana_tasheva@yahoo.com
}

The contraindications of the physiotherapy in the early phase of recovery were determined in our article (7-9). The physiotherapy program was developed according to muscles dysfunction, limited hip abduction, compensatory lumbar scoliosis, postural reeducation and plyometry training.

THE PURPOSE of this study is to present the functional diagnostic in one of the most common compensatory syndromes, namely "short leg", in patients after surgically treated complex acetabular fractures, and to control it by physiotherapy.

\section{METHODS}

The observed 22 patients, 7 female and 15 male, from February 2000 until June 2019 were with dislocated complex acetabular fractures in multiple traumas (Figure 1). Their average age was 49,5 years. The surgical stabilized method with reconstructive plates and screws was applied (2-4) (Figure 2). 


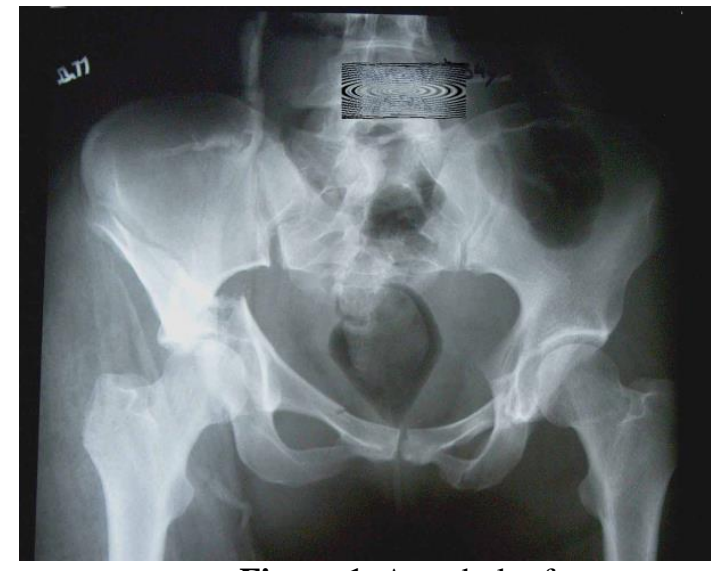

Figure 1. Acetabular fracture

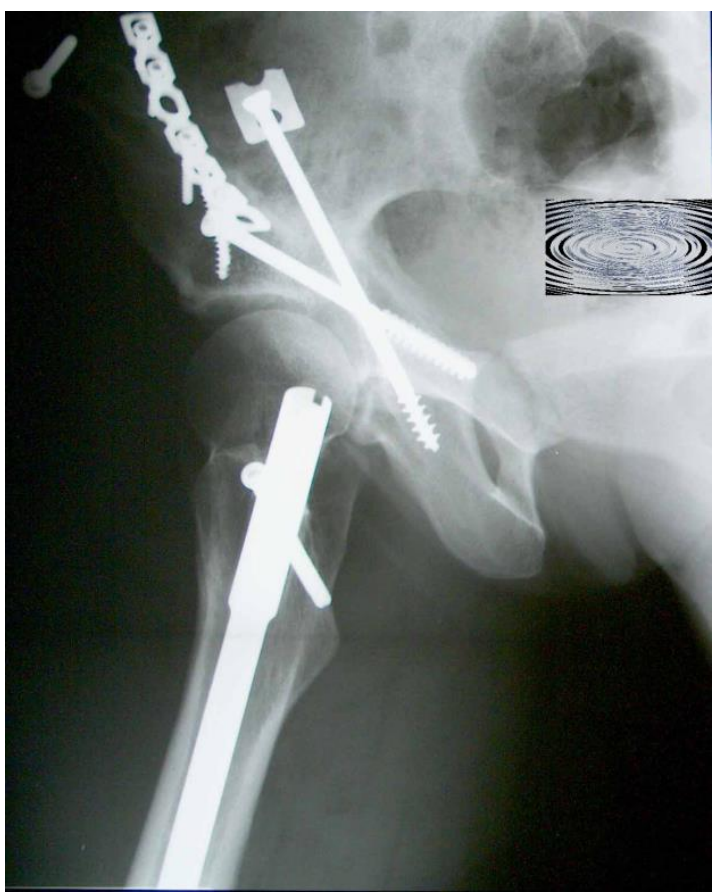

Figure 2. Surgical stabilized acetabular fracture

Fourteen of the patients were from car crash and one from motorcycle accidents, and 3 athletes in hang gliding and 4 in motoring.

The multiple traumas, direct extension, surgical intervention and walking with aids (crutches and walker) provoke a vestibular and proprioceptive adaptation (Figures $3, \mathbf{4}$ ).

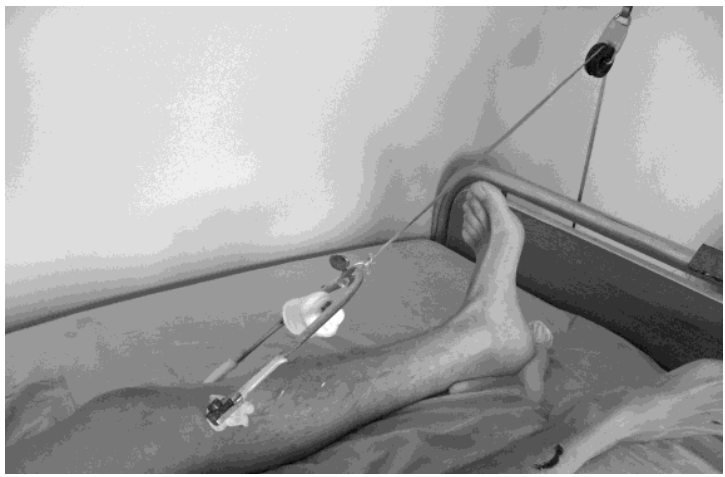

Figure 3. Direct extension

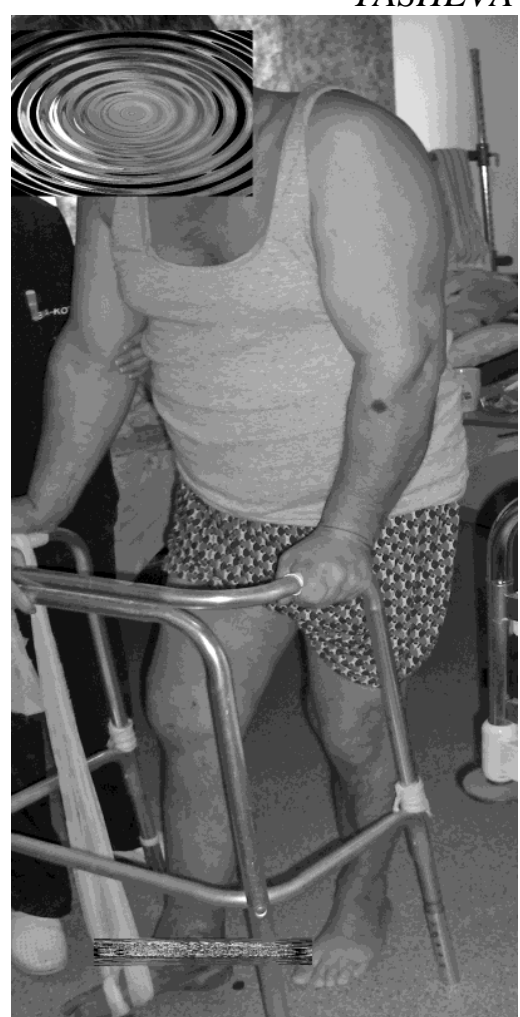

Figure 4. Walking with a walker

A "short leg" syndrome is a typical effect of this adaptation (Figures 5, 6). The dysfunction frequently presents without any requirements for treatment in practice. However the symptoms affect back, contralateral lower limb and whole body.

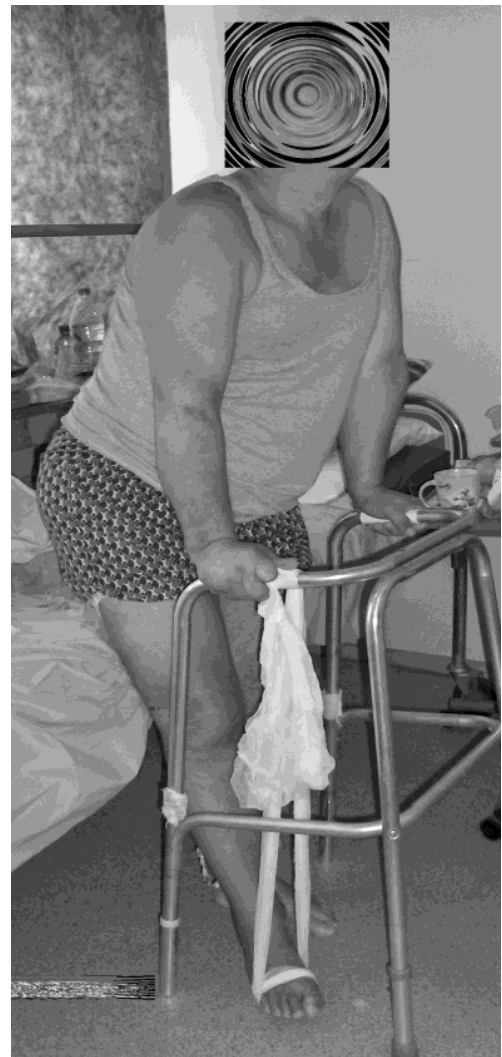

Figure 5. "Short leg" in standing 


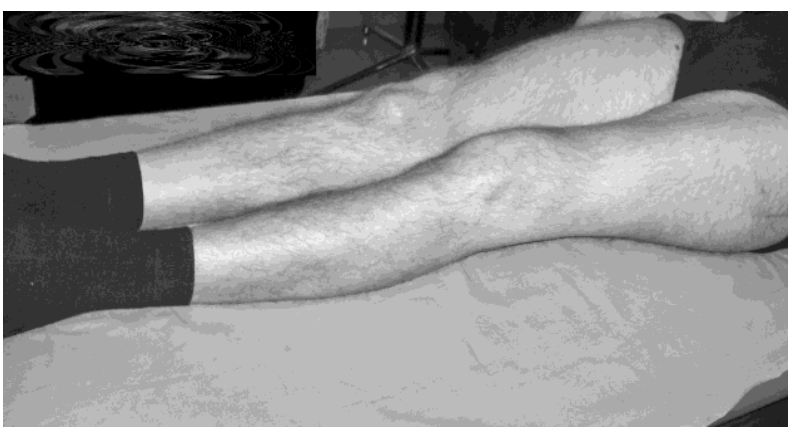

Figure 6. "Short leg" in supine

The examination of the patients included: alignment of both anterior superior spines, spine, levels of iliac crests, hip-to-ankle measurements, medial malleolus levels, greater trochanter heights, shoulders level, soft tissue stress and tightness.

The physiotherapy program aims to avoid this compensatory mechanism by controlling anterior and lateral pelvis tilt and rotation, lumbar scoliosis, and soft tissue imbalance.

The correct treatment by physiotherapy needs the precious diagnostic by visual, palpation and functional assessment. The compensation of the short leg leads to lumbar scoliosis away from and pelvis rotation toward the short leg. The lumbosacral angle is increased. The patients rotate the pelvis anterior to lengthen the contralateral leg. The change of the apparent long leg is foot pronation. The lumbar scoliosis causes shortening the concave erector spinae, quadratus lumborum and hamstrings.

Pelvis stabilization was achieved by physiotherapy modalities such as unilateral relaxation of hamstrings, erector spinae, multifidus and quadratus lumborum, and their contralateral stimulation (Figures 7, 8).

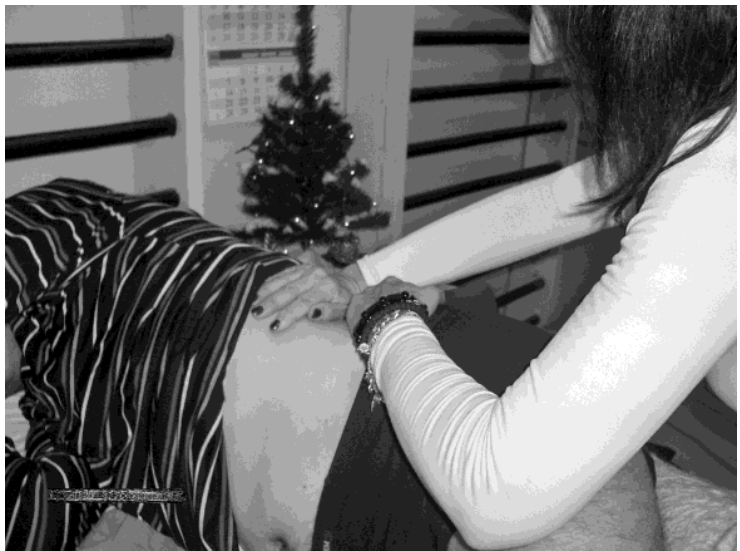

Figure 7. M.quadratus lumborum' relaxation

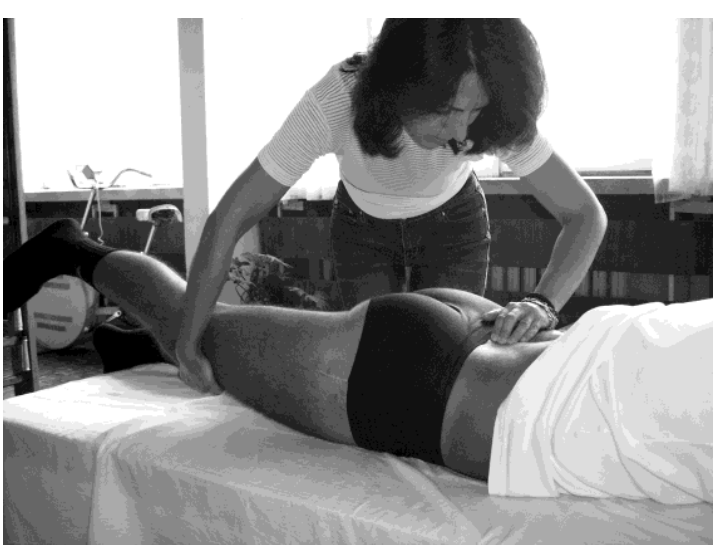

Figure 8. M.erector spinae' relaxation

The physiotherapy progressed by the relaxation of the hip adductors and stimulation of the hip abductor on the operated lower limb side (Figures 9, 10).

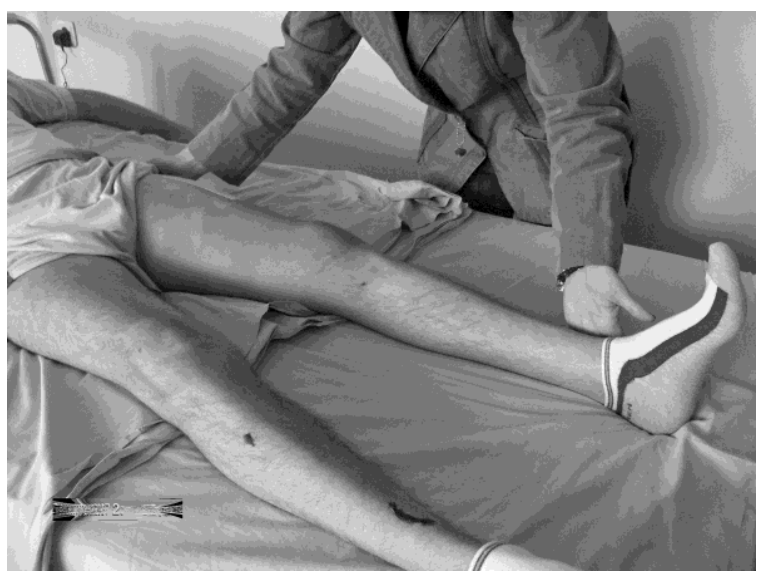

Figure 9. Stimulation of the abductor

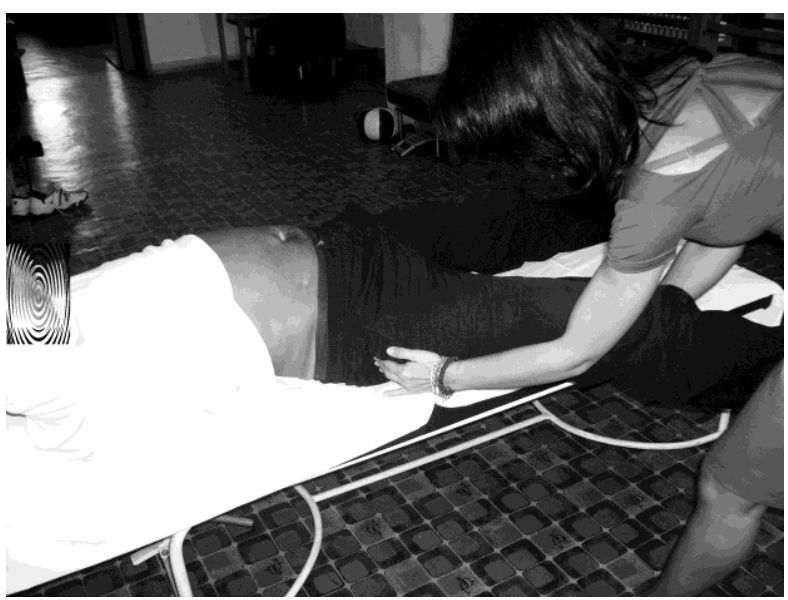

Figure 10. Two points abductors' stimulation

The post isometric relaxation of the hamstrings on one side and the. m. quadriceps femoris on the other, and their contralateral strengthening was the next step (Figure 11, 12). M. iliopsoas was relaxed on the both sides. 


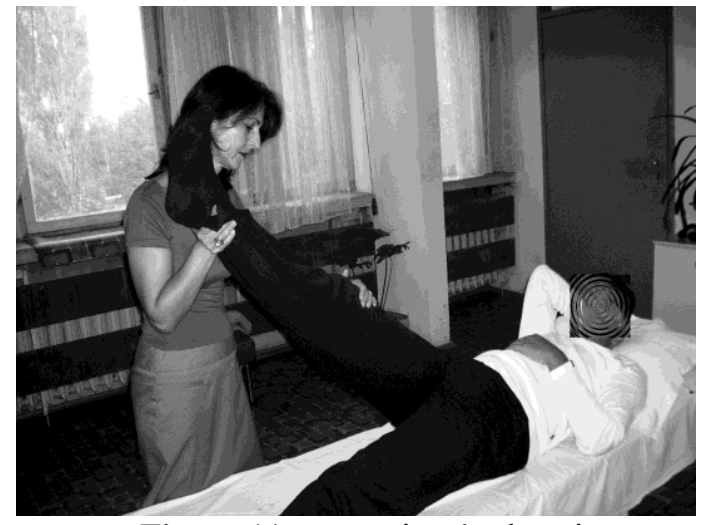

Figure 11. Hamstrings' relaxation

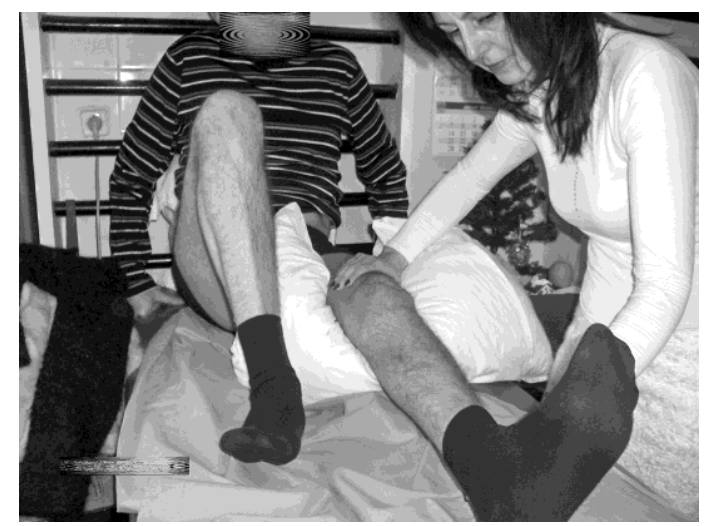

Figure 12. M. quadriceps femoris' stimulation

The procedure involved combination of pain relief techniques, mobilization and relaxation of the iliolumbar and sacroiliac ligaments. Dysfunctional patterns affect the postural muscles and it is necessary to educate patients to control pelvis, to flex knee, to strike and rock the heel (Figure 13). Correct walking was accomplished by avoiding the pronated foot on the no operated leg and the heel lift on the operated one (Figure 14).

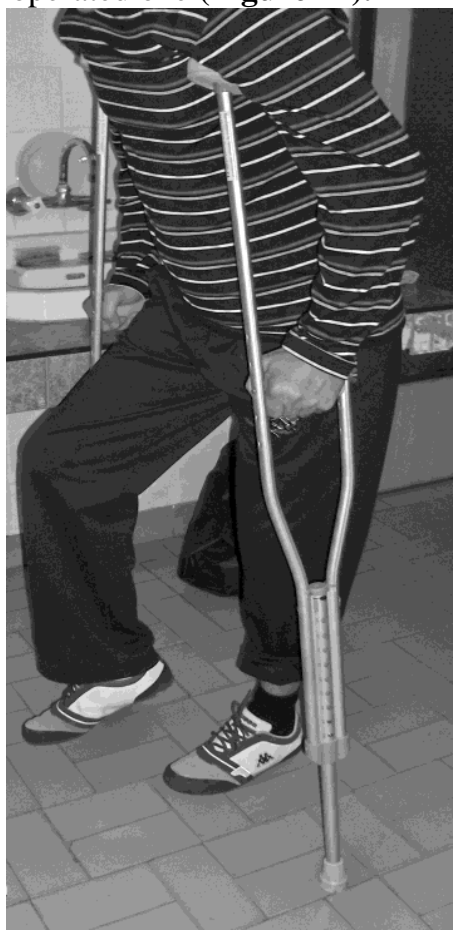

Figure13. Walking with knee flexion

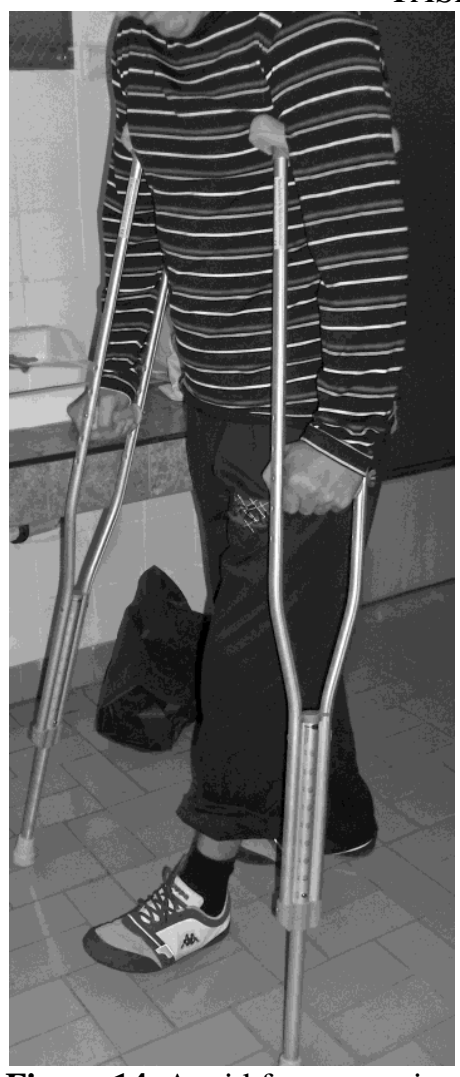

Figure 14. Avoid foot pronation

\section{RESULTS}

The compensation efficiency varies from patient to patient and this problem has to precisely identify. The results of the examination of the patients showed out of alignment of the next indicators: procesus spinosus, both anterior superior spines, levels of iliac crests, medial malleolus levels, greater trochanter heights, shoulders levels.

There were differences between greater trochanter-to-ankle and spina iliaca anterior superior-to-ankle measurements with average $1,7 \mathrm{~cm}$. Apart of the complex symptoms the standing flexion test was positive. By the chosen methods of diagnostic also were found the unilateral shorten tensor fascia latae, adductors, hamstrings and quadriceps. $M$. iliopsoas was shortened bilateral.

Compensatory lumbar scoliosis was diagnosed on the contralateral side.

Following the developed physiotherapy techniques such as pain relief, muscles energy techniques and gait control the results of the tests were negative at the end of the relative protection phase of recovery for 7 procedures. 


\section{DISCUSSION}

Optimal recovery was achieved through reducing of the soft tissue stress and tightness. Management of lumbar scoliosis and restoring the alignment of both anterior superior spines, levels of iliac crests, hip-to-ankle distances, medial malleolus levels, greater trochanter heights, shoulders levels, are the leading factors in preventing complications in time.

Variety of the chosen methods gave correct and adequate information about changes of the passive and dynamic tissues. Depending on the continuance of the symptoms the imbalance usually related to stress of the iliolumbar and sacroiliac ligaments on the convexity side. The pain referred from the first one to the ipsilateral groin and upper medial thigh, and from the second one into the lateral leg. Applied mobilization and soft tissue reduced the symptoms.

\section{CONCLUSION}

The social importance of physiotherapy is not only to restore correct and optimal patients, but also to provide prevention of complications after surgically stabilized complex acetabular fractures through avoid the compensatory mechanisms and dysfunction. One of them is a "short leg" syndrome which is overcome by applied individual criteria for diagnostic and treatment by physiotherapy modalities.

\section{REFERENCES}

1. Iotov, A., Enchev, D., Tcachev, N., Osteosynthesis in fractures. In: Tivchev, P., Kinov, Pl., Hip surgery. Bgbook, Sofia: 592-668, 2016.
TASHEVA $R$.

2. Iotov, A. Surgical approaches with surgical treatment of acetabular fracture.

Emergency Medicine, 2: 14- 21, 2000.

3. Iotov, A., et al., Limited invasive surgical technique for some complex acetabular fractures. Bulg J Orthop trauma, 2(37): 8187, 2001.

4. Borrelli, J., et al., Functional outcome after isolated acetabular fractures. J Ortop Trauma, 2: 73- 81, 2002.

5. Kuchera, M., Kuchera, W., Postural considerations in coronal and horizontal planes. In: Ward R. Foundations for osteopathic medicine. Williams\&Walkins, Baltimore, 1997.

6. Steindlern, A., Kinesiology of the human body /on the mechanics of gait/. II edit., $631-691,1974$.

7. Quadagno, J., Why the United States has no national health insurance. $J$ Health and Social Behavior, 45: 25-44, 2004.

8. Tasheva, R., Milenkova, M., Iotov, A., et al., Physiotherapy behavior in the early stage of surgically treated complex acetabular fractures. Scientific Conference with International Participation on Problems of Human Motion. Silistra, 2- $4^{\text {th }}$ May: 89-94, 2003.

9. Tasheva, R., Milenkova, M., Iotov, A., et al., Physiotherapy Algorithm after Surgical Treatment of the Paragliding Multiplan Trauma. Bulg J Kines \& Reh, 1-2: 46-51, 2004.

10.Tasheva, R., The effect of motor control physiotherapy after a hang glider's surgically treated multiple traumas. J Sports Med Assoc of Greece, December, V1, Issue 1: 20-23, 2006. 